\title{
056 IS COGNITIVE SCREENING IN PRIMARY CARE
} CHANGING?

Mona Ghadiri-Sani, AJ Larner. Walton Centre for Neurology \& Neurosurgery

10.1136/jnnp-2014-309236.56

Objective To examine frequency of cognitive screening instrument (CSI) use in primary care referrals to a cognitive clinic and compare with previous cohorts.

Results Of 185 consecutive new outpatients (July-December 2013), 140 referrals $(75.7 \%)$ were from primary care; 44 (31.4\%) had evidence for CSI use based on the referral letter. In previous cohorts from primary care CSI frequencies were 20.9\% (Oct 2004-Sept 2006), 25.9\% (Feb 2008-Feb 2009), and 26.8\% (Feb 2009-Feb 2010). The null hypothesis that the proportion of CSI use in primary care did not differ significantly in sequential cohorts was not rejected $(\chi 2=3.94, \mathrm{df}=3$, $\mathrm{p}>0.1)$. However, there was an increase in the proportion of patients administered newer CSI suitable for primary care use (6CIT, GPCOG) compared to older CSI (MMSE, AMTS) in the 2013 cohort ( 0.48 vs 0.43 ) compared to sum of the previous 3 cohorts (0.04 vs 0.91$)$.

Conclusions Although there has been no overall increase in CSI use in primary care referrals, the use of CSI appropriate for primary care use is increasing. 